\title{
Effect of the space charge layer created by corona at ground level on the inception of upward lightning leaders from tall towers
}

\author{
M. Becerra, ${ }^{1}$ V. Cooray, ${ }^{1}$ S. Soula, ${ }^{2}$ and S. Chauzy ${ }^{2}$ \\ Received 2 December 2006; revised 26 February 2007; accepted 3 April 2007; published 20 June 2007.
}

[1] Electric field measurements above ground have shown that the space charge layer created by corona at ground level shields the background electric field produced by the thundercloud. Therefore it is expected that this space charge layer can also influence the conditions required to initiate upward lightning from tall objects. For this reason, a numerical model that describes the evolution of the main electrical parameters below a thunderstorm is used to compute the space charge layer development. The time variation of the electric field measured at $600 \mathrm{~m}$ above ground during the 1989 rocket triggered lightning experiment at the Kennedy Space Center (Florida) is used to drive the model. The obtained space charge density profiles are used to compute the conditions required to initiate stable upward lightning positive leaders from tall towers. Corona at the tip of the tower is neglected. It is found that the space charge layer significantly affects the critical thundercloud electric fields required to initiate upward lightning leaders from tall objects. The neutral aerosol particle concentration is observed to have a significant influence on the space charge density profiles and the critical thundercloud electric fields, whereas the corona current density does not considerably affect the results for the cases considered in the analysis. It is found that a lower thundercloud electric field is required to trigger a lightning flash from a tall tower or other tall slender grounded structure in the case of sites with a high neutral aerosol particle concentration, like polluted areas or coastal regions.

Citation: Becerra, M., V. Cooray, S. Soula, and S. Chauzy (2007), Effect of the space charge layer created by corona at ground level on the inception of upward lightning leaders from tall towers, J. Geophys. Res., 112, D12205, doi:10.1029/2006JD008308.

\section{Introduction}

[2] Low-altitude soundings [Standler and Winn, 1979; Chauzy and Raizonville, 1982] have shown that the electric fields underneath thunderclouds are significantly reduced by the shielding effect of the space charge layer formed by ions produced by corona at ground level. The electric fields at the ground usually do not exceed $10 \mathrm{kV} \mathrm{m}^{-1}$ even though the thundercloud electric fields aloft can be several times larger [Soula and Chauzy, 1991]. This electric field shielding produced by the space charge layer extends in altitude up to several hundreds of meters above ground [Willet et al., 1999; Qie et al., 1994] depending mainly upon the neutral aerosol particle concentration of the site [Chauzy and Raizonville, 1982; Qie et al., 1994].

[3] Upward lightning from tall grounded objects is triggered by the ambient electric field at the top of them [Berger, 1977]. This ambient electric field is initially produced by the thundercloud charges and modified by

\footnotetext{
${ }^{1}$ Division of Electricity and Lightning Research, Uppsala University, Uppsala, Sweden.

${ }^{2}$ Laboratoire d'Aérologie, UMR CNRS, Université Paul Sabatier, Toulouse, France.
}

the space charge layer created around these objects. This charge layer can therefore change the necessary conditions to initiate upward leaders from tall objects [Dellera et al., 1997]. Hence, Golde [1978] qualitatively explained the differences between the photographs of lightning strikes to tall towers in Switzerland and in South Africa in terms of the shielding effect produced by the space charge layer. According to Golde [1978] "the masts in San Salvatore, or any other tower surrounded by forests or other tall structures would in consequence act similarly to a low building when subjected to the field of an approaching leader... in contrast to an isolated mast, like that near of Pretoria."

[4] However, a specific and thorough quantitative analysis of the effect of the space charge layer, created by corona on a large surface at ground level, on the initiation of self-propagating upward leaders from grounded structures cannot be found in the current literature. Even though intensive research has been performed on the effect of corona at the tip of grounded objects on leader initiation [Aleksandrov et al., 2001, 2002, 2005], it relates only to the local space charge created in the vicinity of the object tip. This space charge is created in a small volume around the object tip when the electric field locally exceeds about $3 \mathrm{MV} \mathrm{m}{ }^{-1}$, reaching densities larger than $10^{-6} \mathrm{C} \mathrm{m}^{-3}$ 
[Aleksandrov et al., 2002]. This case is different from the space charge layer created by corona at ground level by irregularities on the surface (grass, bushes, trees,...etc), which is initiated when the electric fields at the ground surface reach few kilovolts per meter and can reach densities in the order of $10^{-9} \mathrm{C} \mathrm{m}^{-3}$ close to ground [Chauzy and Raizonville, 1982].

[5] A brief analysis of the influence of the space charge layer on the ground electric field required to initiate stable upward positive leaders was addressed in the discussion in the work of Rizk [1994]. It was merely mentioned that the effect of the space charge layer on leader inception conditions can be important and that this effect might be less significant because of rain, wind, and the shielding effect of the structure. In addition, Sunaga and Shindo [2005] showed that the space charge layer could substantially distort the electric field around conductors in a transmission line and that this effect can influence the initiation of upward leaders.

[6] The present paper is devoted to a numerical analysis of the space charge layer created by corona at ground level around tall towers and its effect on the inception of upward lightning leaders under the influence of the thundercloud electric field. A two-dimensional model is used to evaluate the drift of the ions in the space charge layer. In order to consider a realistic case, the space charge calculation is driven by the thundercloud electric field measured at $600 \mathrm{~m}$ above ground, during a thunderstorm in Florida (USA), on 10 August 1989 [Soula and Chauzy, 1991]. In this experiment, the multilevel electric field measuring system was located aloft using a tethered balloon during a thunderstorm at the Kennedy Space Center. In this way, the electric field was measured at ground level and at 80, 436, 600, and $800 \mathrm{~m}$ above ground, during the charging process of the thundercloud. During the period of the field measurement, several cloud-to-ground (CG) lightning flashes were triggered by rocket technique. From these data, the space charge density profiles present at the moment of the triggered CG flashes are computed and used to evaluate the critical thundercloud electric fields required to initiate stable upward leaders from isolated towers located on flat terrain. We discuss the influence of various features of a particular site, such as the corona current density at ground and the neutral aerosol particle concentration, on the space charge layer and the critical thundercloud electric fields necessary to initiate upward leaders.

\section{Space Charge Numerical Modeling}

\subsection{Numerical Algorithm}

[7] In this work, the one-dimensional model proposed by Chauzy and Renella [1985] is extended to two dimensions. A system of cylindrical coordinates is used to consider the axial and radial drift of the ions in the electric field produced by the thundercloud taking into account the reduction of the ground field produced by the tower. Then, the continuity equations for small positive ions $n_{+}$, positively charged aerosol particles (large positive ions) $N_{+}$, small negative ions $n_{-}$, negative charged aerosol particles (large negative ions) $N_{-}$, and neutral aerosol particles $N_{0}$ :

$$
\begin{aligned}
\frac{\partial n_{+}}{\partial t}= & -k_{n+N o} \cdot n_{+} \cdot N_{0}-k_{n+N-} \cdot n_{+} \cdot N_{-}-\alpha \cdot n_{+} \cdot n_{-} \\
& -\nabla \cdot\left(n_{+} \cdot \mu_{n+} \cdot \bar{E}\right)+D \cdot \nabla^{2} n_{+}
\end{aligned}
$$

$$
\begin{aligned}
\frac{\partial N_{+}}{\partial t}= & k_{n+N_{0}} \cdot n_{+} \cdot N_{0}-k_{n-N+} \cdot n_{-} \cdot N_{+} \\
& -\nabla \cdot\left(N_{+} \cdot \mu_{N+} \cdot \bar{E}\right)+D \cdot \nabla^{2} N_{+} \\
\frac{\partial n_{-}}{\partial t}= & -k_{n-N_{0}} \cdot n_{-} \cdot N_{0}-k_{n-N+} \cdot n_{-} \cdot N_{+}-\alpha \cdot n_{+} \cdot n_{-} \\
& -\nabla \cdot\left(n_{-} \cdot \mu_{n-} \cdot \bar{E}\right)+D \cdot \nabla^{2} n_{-}
\end{aligned}
$$

$$
\begin{aligned}
\frac{\partial N_{-}}{\partial t}= & k_{n-N_{0}} \cdot n_{-} \cdot N_{0}-k_{n+N_{-}} \cdot n_{+} \cdot N_{-} \\
& -\nabla \cdot\left(N_{-} \cdot \mu_{N-} \cdot \bar{E}\right)+D \cdot \nabla^{2} N_{-} \\
\frac{\partial N_{0}}{\partial t}= & -k_{n+N_{0}} \cdot n_{+} \cdot N_{0}-k_{n-N_{0}} \cdot n_{-} \cdot N_{0} \\
& +k_{n-N+} \cdot n_{-} \cdot N_{+}+k_{n+N-} \cdot n_{+} \cdot N_{-}-D \cdot \nabla^{2} N_{0}
\end{aligned}
$$

are solved together with the Poisson's equation for electric field $\bar{E}$ and potential $\Phi$ :

$$
\nabla \cdot \bar{E}=-\nabla^{2} \Phi=\frac{e \cdot\left(n_{+}+N_{+}-n_{-}-N_{-}\right)}{\varepsilon_{0}}
$$

where $\mu$ is the mobility of the charged particles, with values of $\mu_{n^{+}}=1.5 \times 10^{-4} \mathrm{~m}^{2} \mathrm{~s}^{-1} \mathrm{~V}^{-1}$ for small positive ions, $\mu_{N^{+}}=1.5 \times 10^{-6} \mathrm{~m}^{2} \mathrm{~s}^{-1} \mathrm{~V}^{-1}$ for large positive ions, $\mu_{n-}=$ $-2 \times 10^{-4} \mathrm{~m}^{2} \mathrm{~s}^{-1} \mathrm{~V}^{-1}$ for small negative ions and $\mu_{N-}=$ $-2 \times 10^{-6} \mathrm{~m}^{2} \mathrm{~s}^{-1} \mathrm{~V}^{-1}$ for large negative ions. The attachment coefficients of the small positive and negative ions to neutral aerosol particles $k_{n+N_{0}}$ and $k_{n-N_{0}}$ are equal to $2.9 \times 10^{-12} \mathrm{~m}^{3} \mathrm{~s}^{-1}$ and $3.5 \times 10^{-12} \mathrm{~m}^{3} \mathrm{~s}^{-1^{0}}$ respectively. The recombination coefficient of small ions with opposite polarity $\alpha$ is equal to $1.6 \times 10^{-12} \mathrm{~m}^{3} \mathrm{~s}^{-1}$, while the recombination coefficients of small positive ions to negative large ions $k_{n+N-}$ and of small negative ions to positively charged aerosol particles $k_{n-N^{+}}$are set as $5 \times 10^{-12} \mathrm{~m}^{3} \mathrm{~s}^{-1}$ and $6 \times 10^{-12} \mathrm{~m}^{3} \mathrm{~s}^{-1}$ respectively. The value of the turbulent diffusion coefficient $D$ is chosen as $1 \mathrm{~m}^{2} \mathrm{~s}^{-1}$. The values used for these quantities are taken from the work of Qie et al. [1994]. $\varepsilon_{0}$ is the dielectric permittivity of vacuum, and $e$ is the elementary charge.

[8] The transport terms in equations (1)-(5) are solved with a finite difference control volume approach [Roache, 1976], and the source terms in the continuity equations are included with a second order Runge-Kutta scheme. For sake of simplicity, any extra term to account for the effect of wind in the drift of the charged particles is disregarded. The Poisson's equation is solved by considering separately the contribution of both the tower/thundercloud and the formed space charge layer. Hence the Optimized Charge Simulation 
Method [Yializis et al., 1978] is used to compute the background electric fields produced by the thundercloud alone while the distortion produced by the space charge is separately computed with the subprogram SEPELI [Swarztrauber and Sweet, 1975]. In this case, a fourth-order generalized cyclic reduction algorithm is used to solve the Poisson's equation for potential. The electric fields are obtained by calculating the gradient of the potential solution with a second-order finite difference scheme.

\subsection{Boundary Conditions}

[9] A cylindrical coordinate system with axial symmetry $(r, z)$ is used to simulate the space charge drift around an isolated tower located on flat terrain. Thus, an analysis domain is defined from ground $z_{0}$ to the cloud center $z_{1}$ (taken as $4000 \mathrm{~m}$ ) and in the radial direction from the axis of symmetry $r_{0}$ to a finite boundary $r_{1}$. A uniformly spaced computational grid is used. Because of the fact that the size of the computation domain is a limiting factor, the boundary $r_{1}$ is set far enough to approximately satisfy the following condition:

$$
\frac{\partial \Phi\left(r_{1}, z\right)}{\partial r} \cong 0
$$

The smallest value of $r_{1}$ that roughly satisfies the condition (7) is about two to three times the height of the tower, where the electrostatic shielding effect of the tower and the transport of ions in the radial direction become negligible. The spatial resolution of the computational grid is set equal to 2 and $4 \mathrm{~m}$ in the axial and radial directions, respectively.

[10] The boundary conditions for the electrostatic calculation are defined as follows. In order to compute the background electric field produced by the cloud alone, the cloud center of charge is represented by an infinite plane with potential $\Phi\left(r, z_{1}\right)$ and the tower is modeled as a hemispherically capped rod. In this case, the optimized charge simulation method [Yializis et al., 1978] with image charges is used, which defines the ground potential as $\Phi\left(r, z_{0}\right)=0$. The cloud potential $\Phi\left(r, z_{1}\right)$ is set such that the background electric field $E_{\text {cloud }}(t)$ produced by the thundercloud alone is equal to the sum of the electric field $E_{600 \mathrm{~m}}(t)$ measured at $600 \mathrm{~m}$ above ground by Soula and Chauzy [1991] and the space charge electric field at the same altitude. For the calculation of the electric fields produced by the space charges, the same computational domain described above is used. A Neumann boundary condition is then set at $r_{0}$ while the potential along the radial boundary $r_{1}$ is computed by solving the Poisson equation in one dimension with an auxiliary routine that uses a second-order finite difference scheme. During each simulation step, the cloud potential $\Phi\left(r, z_{1}\right)$ is updated with the corresponding measured field $E_{600 \mathrm{~m}}(t)$, and the potential on the tower is evaluated including the induced potentials produced by the space charge layer.

[11] On the other side, for the solution of the continuity equations (1)-(5), small ions are created from the ground surface $z_{0}$ with a current density given by [Soula, 1994]:

$$
J_{\mathrm{c}}=a_{1} \cdot\left(E-E_{0}\right)^{3}+a_{2} \cdot\left(E-E_{0}\right)^{2}+a_{3} \cdot\left(E-E_{0}\right)
$$

where $E$ is the computed ground field, $E_{0}$ is the corona onset field (chosen as $1400 \mathrm{~V} \mathrm{~m}^{-1}$ ) and $a_{1}, a_{2}$, and $a_{3}$ are fitting constants with values of $3.1 \times 10^{-20} \mathrm{~A} \mathrm{~V}^{-3}, 10^{-22} \mathrm{~A} \mathrm{~V}^{-2}$, and 0 , respectively. These values correspond to the first solution used by Qie et al. [1994]. Furthermore, in order to account for the fact that the ions are captured by droplets at the cloud base, it is assumed that all the small ions above the cloud base (defined at a height of $1.5 \mathrm{~km}$ above ground) are converted into large ions.

\section{Evaluation of the Leader Inception Condition}

[12] In order to initiate an upward moving positive leader from any grounded structure under the influence of the electric field produced by a dominant negative cloud charge (upward negative lightning) or because of the combined action of a dominant negative cloud charge and a negative downward stepped leader (downward negative lightning), three main physical conditions have to be fulfilled:

[13] 1. A streamer corona discharge is formed at the tip of the structure (first corona inception).

[14] 2. The stem of the streamer is transformed into a thermalized leader channel (unstable leader inception).

[15] 3. The positive leader channel starts extending and the leader propagation becomes self-sustained (stable leader inception).

[16] Since the initiation of streamer corona discharge from tall towers usually occurs before an unstable leader is incepted (condition satisfied for towers with tip radii smaller than some tens of centimeters), the inception of positive upward leaders is mostly determined by the last two conditions. Therefore the unstable and stable positive leader inception is evaluated in the presence of the space charge layer with the model recently introduced by Becerra and Cooray [2006a]. This model is based on a physical simulation of the first few meters of propagation of an upward positive leader from structures with and without axial symmetry. The simulation of the leader propagation is conducted through an iterative geometrical analysis of the potential distribution in front of the tower tip. Then, it assumes that a stable leader is incepted only if the background electric fields are high enough for the self-sustained propagation of the positive leader at least during the first few meters. The predictions of this model are in excellent agreement with the results of the triggered lightning experiment of Willet et al. [1999] when the space charge left behind the rocket is included. Because of the fact that the model of Becerra and Cooray [2006a] particularly applies to slowly changing background fields as in the case of upward initiated flashes from tall towers and triggered lightning experiments [Becerra and Cooray, 2006b], the results presented in this paper are restricted to upward initiated negative lightning (positive upward leader, negative cloud charge, negative continuous flash) from tall towers or triggering rockets. However, the local space charge produced by corona at the tip of the tower is not analyzed in this paper. Hence the predictions of the present model provide correct values of the background electric field necessary to launch leaders only from triggering rockets or tall towers whose tip radius is large enough so that the corona produced at the tower tip can be neglected. Otherwise, our calculations only provide a lower limit because of the fact that the actual background electric field necessary to initiate an upward leader in the presence of glow 


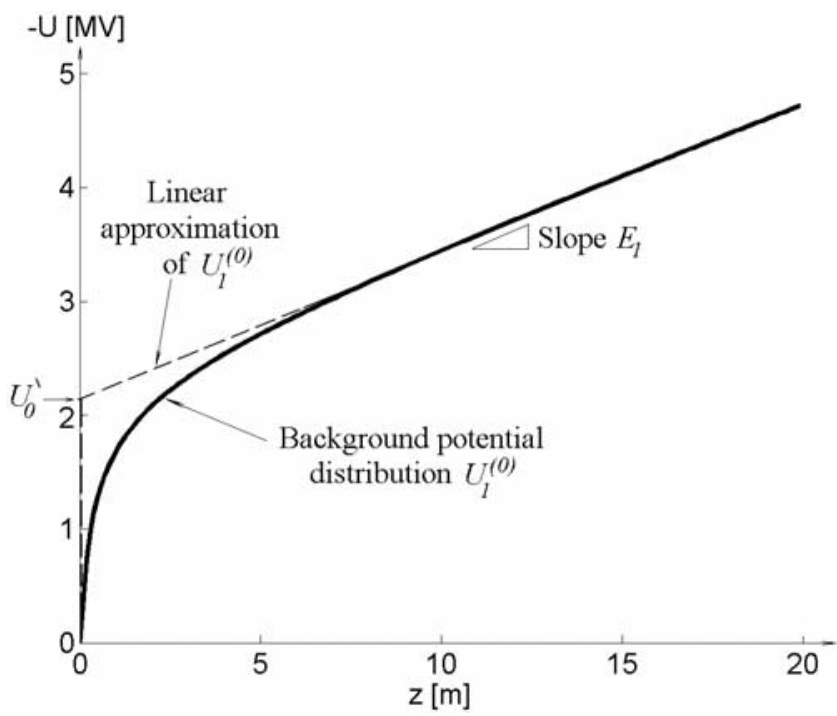

Figure 1. Example of the linear approximation of the background potential distribution along a vertical line from the tip of a 100-m-tall tower under the influence of a thundercloud electric field of $20 \mathrm{kV} \mathrm{m}^{-1}$.

corona from the tower tip could be larger than the present estimated values. For further information on the effect of this space charge formed in the vicinity of the tower tip on the inception of upward leaders, the reader is referred to Aleksandrov et al. [2001, 2002, 2005].

[17] In this paper, the following procedure is used to determine whether a self-propagating upward leader is incepted or not from a grounded tall structure under a given thundercloud field:

[18] 1. The background potential distribution $U_{1}$ produced by the thundercloud electric field and the space charge in front of the tower's tip is computed. This potential $U_{1}$ is calculated along a vertical line from the tower's tip utilizing the charge simulation method and taking into account the potential produced by the space charge layer. It corresponds to the potential along the axis of symmetry such that $U_{1}\left(z^{\prime}\right)=\Phi\left(0, z_{\text {tip }}+z^{\prime}\right)$ where $z_{\text {tip }}$ is the height of the tower. In the analysis, the potential distribution $U_{1}\left(z^{\prime}\right)$ is approximated by a straight line with slope $E_{1}$ and intercept $U_{0}^{\prime}$, as shown in Figure 1:

$$
U_{1}^{(0)}\left(z^{\prime}\right) \approx E_{1} \cdot z^{\prime}+U_{0}^{\prime}
$$

[19] 2. The charge $\Delta Q^{(0)}$ and position $l_{\mathrm{s}}^{(0)}$ of the streamer corona are computed as

$$
\begin{gathered}
\Delta Q^{(0)} \approx K_{Q} \cdot \frac{U_{0}^{\prime 2}}{2 \cdot\left(E_{\mathrm{str}}-E_{1}\right)} \\
l_{\mathrm{s}}^{(0)}=\frac{U_{0}^{\prime}}{E_{\mathrm{str}}-E_{1}}
\end{gathered}
$$

where $K_{Q}$ is a geometrical factor and $E_{\mathrm{str}}$ is the positive streamer gradient. If the charge of the corona burst $\Delta Q^{(0)}$ is lower than $1 \mu \mathrm{C}$, the unstable leader inception condition is not fulfilled and the analysis stops. Otherwise, the simulation of the leader propagation starts $i=1$ with an initial leader length $l_{\mathrm{L}}^{(1)}$.

[20] 3. The potential at the leader's tip $U_{\text {tip }}(i)$ during the ith step of the simulation is computed as:

$$
U_{\text {tip }}^{(i)}=l_{\mathrm{L}}^{(i)} \cdot E_{\infty}+x_{0} \cdot E_{\infty} \cdot \ln \left[\frac{E_{\text {str }}}{E_{\infty}}-\frac{E_{\text {str }}-E_{\infty}}{E_{\infty}} \cdot e^{-l_{\mathrm{L}}^{(i) / x_{0}}}\right]
$$

where $l_{\mathrm{L}}^{(i)}$ is the leader length at the $i$ th simulation step, $E_{\infty}$ is the final quasi-stationary leader gradient, $x_{0}$ is a constant given by the product of the ascending positive leader speed and the leader time constant.

[21] 4. The position and charge of the corona zone at the leader tip are calculated as:

$$
l_{\mathrm{s}}^{(i)}=l_{\mathrm{s}}^{(0)}+\frac{E_{\mathrm{str}} \cdot l_{\mathrm{L}}^{(i)}-U_{\mathrm{tip}}^{(i)}}{E_{\mathrm{str}}-E_{1}}
$$

$$
\begin{aligned}
\Delta Q^{(i)} \approx K_{Q} \cdot & \left\{\left(E_{\mathrm{str}} \cdot\left(l_{\mathrm{L}}^{(i)}-l_{\mathrm{L}}^{(i-1)}\right)+U_{\text {tip }}^{(i-1)}-U_{\text {tip }}^{(i)}\right)\right. \\
\cdot & \left.\left(l_{\mathrm{s}}^{(i-1)}-l_{\mathrm{L}}^{(i)}\right)\right\}
\end{aligned}
$$

[22] 5. The leader advancement distance $\Delta l_{\mathrm{L}}^{(i)}$ and the new leader length $l_{\mathrm{L}}^{(i+1)}$ are computed as:

$$
\begin{gathered}
\Delta l_{\mathrm{L}}^{(i)}=\frac{\Delta Q^{(i)}}{q_{\mathrm{L}}} \\
l_{\mathrm{L}}^{(i+1)}=l_{\mathrm{L}}^{(i)}+\Delta l_{\mathrm{L}}^{(i)}
\end{gathered}
$$

If the leader length $l_{\mathrm{L}}^{(i+1)}$ reaches a maximum value $l_{\text {max }}$, then the stable leader inception condition is fulfilled. If the leader advancement length $\Delta l_{\mathrm{L}}^{(i)}$ starts to decrease after several steps, then the leader stops and the leader inception is not reached. Otherwise, the simulation of the leader propagation continues by going back to 3 . According to the background electric field, either the leader continues to propagate or the value of $\Delta Q^{(i)}$ continues to decrease monotonically leading to the arrest of the leader. In our analysis, a stable leader inception condition is assumed if the leader continues to propagate and the leader length $l_{\mathrm{L}}^{(i+1)}$ reaches the maximum value $l_{\max }$. A typical value of $l_{\max }$ equal to $2 \mathrm{~m}$ was observed to be long enough to define the stable propagation of an upward positive leader. Larger values require more simulation steps $i$ and produce the same result. The values of the physical parameters used in equations (10)-(13) are presented in Table 1. Further details on the chosen values can be found in the work of Becerra and Cooray [2006a].

\section{Results and Discussion}

[23] The corona produced at ground level during thunderstorm conditions can be affected by different factors such as the thundercloud electric field, the neutral aerosol particle concentration, and the kind of vegetation (the structure of the terrain) [Chauzy and Raizonville, 1982]. Also, a tall structure reduces the electric field in its vicinity, inhibiting 
Table 1. Parameters Used for the Evaluation of the Upward Leader Inception Condition

\begin{tabular}{llcc}
\hline Symbol & \multicolumn{1}{c}{ Description } & Value & Unit \\
\hline$l_{\mathrm{L}}^{(1)}$ & Initial leader length & $5 \times 10^{-2}$ & $\mathrm{~m}$ \\
$E_{\text {str }}$ & Positive streamer gradient, equations (10), (11), (12) & $4.5 \times 10^{5}$ & $\mathrm{~V} / \mathrm{m}$ \\
$E_{\infty}$ & Final quasi-stationary leader gradient, equation (11) & $3 \times 10^{4}$ & $\mathrm{~V} / \mathrm{m}$ \\
$x_{0}$ & Constant given by the ascending positive leader & 0.75 & $\mathrm{~m}$ \\
& speed and the leader time constant, equation (11) & $65 \times 10^{-6}$ & $\mathrm{C} / \mathrm{m}$ \\
$q_{\mathrm{L}}$ & Charge per unit length necessary to thermal transition, equation (13a) & $4 \times 10^{-11}$ & $\mathrm{C} / \mathrm{V} \cdot \mathrm{m}$ \\
$K_{Q}$ & Geometrical constant that correlates the potential distribution and the & & \\
\hline
\end{tabular}

corona production. Thus, the effect of these factors on the space charge layer and consequently on the critical thundercloud electric field required to initiate upward leaders from tall towers is discussed in this section.

[24] In order to consider a realistic case, the electric field measured by Soula and Chauzy [1991] at $600 \mathrm{~m}$ above ground during a thunderstorm on 10 August 1989 in Florida, is used to drive the model. During that experiment, the electric field was measured at ground level and aloft during the development of a thundercloud, and four CG triggered lightning flashes were triggered (23:47 (first flash), 23:53 (second flash), 23:58 (third flash), and 00:04 (fourth flash) UT), as shown in Figure 2.

\subsection{Shielding Effect Around the Tower}

[25] To evaluate the effect of the electric field shielding produced by the tower on the corona generation at ground, a 100 -m-tall tower is simulated. Figure 3 shows the space charge density contours at the moment of the first triggered flash (23:47 UT). Note that the shielding of the surface electric field inhibits the corona generation around the tower, reducing significantly the space charge density close to it. Nonetheless, this effect does not extend more than several tens of meters from the tower beyond which the space charge layer develops almost uniformly. In spite of the "hole" in the space charge layer around the tower, the space charge potential along the axis of symmetry (that defines the potential induced by the space charge layer on the tower surface) does not differ significantly from the potential evaluated along a vertical line at any other radial

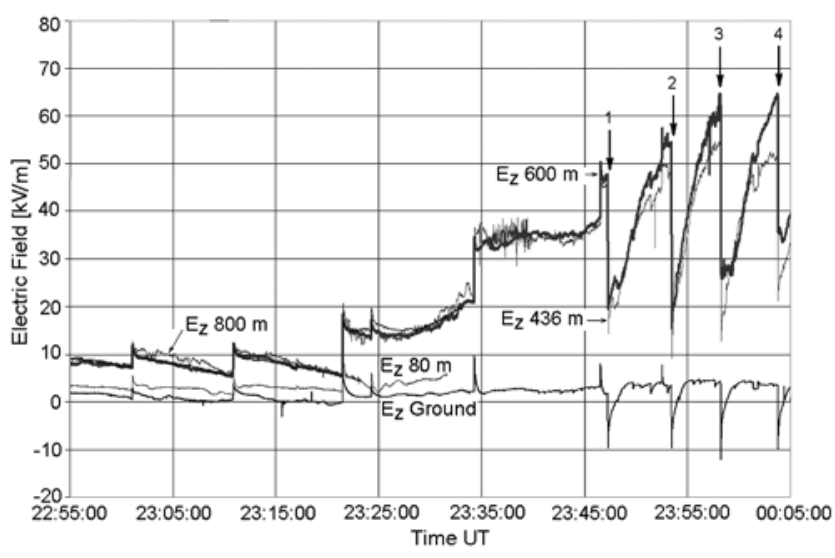

Figure 2. Electric field variations measured at ground and aloft beneath a thunderstorm on 10 August 1989, at Kennedy Space Center [Soula and Chauzy, 1991]. The numbered arrows correspond to the triggered CG flashes. position. This suggests that even though the presence of the tower inhibits the corona formation around it, the potential distribution caused by the space charge layer does not differ much from that produced by a uniform space charge layer. For this reason, the potential distribution of a uniformly developing space charge layer was computed with a onedimensional model and compared to the more exact twodimensional calculation. Differences lower than 5\% were found between the one- and two-dimensional calculations. Thus, given the complexity of the two-dimensional calculation and the small differences found between the onedimensional and two-dimensional models, the space charge analysis presented hereinafter is performed with the onedimensional calculation.

\subsection{Effect of the Neutral Aerosol Particle Concentration}

[26] The concentration of neutral aerosol particles has been observed to have a significant influence on the evolution of the electric field produced by the corona space charge layer [Chauzy and Raizonville, 1982; Qie et al., 1994]. Since the neutral aerosol particle concentration changes drastically from typical values of about $5 \times 10^{9} \mathrm{~m}^{-3}$ for clean rural zones to values exceeding $10^{11} \mathrm{~m}^{-3}$ for urban, polluted places [Twomey, 1977], its influence is an important factor to consider.

[27] The space charge layer is computed for different aerosol concentrations, assuming values used for the south of France $N_{0}=5 \times 10^{9} \mathrm{~m}^{-3}$, Florida $N_{0}=10^{10} \mathrm{~m}^{-3}$ [Chauzy and Soula, 1999], and for a moderately polluted

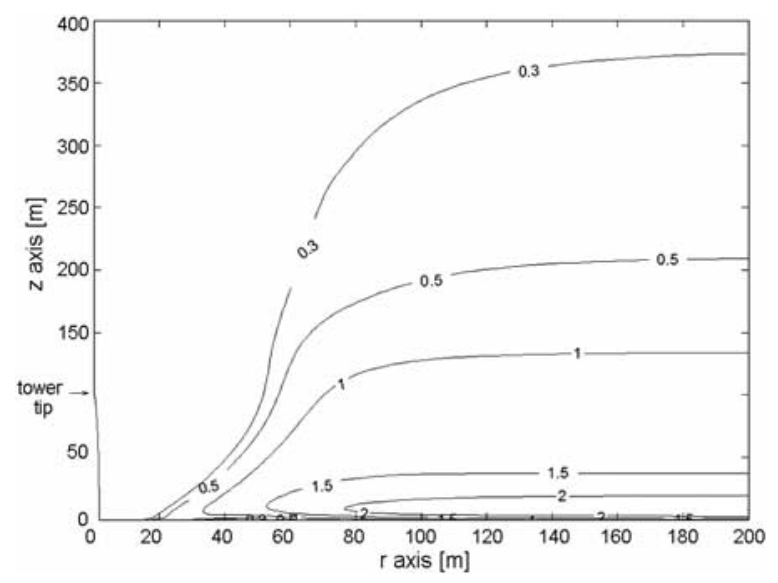

Figure 3. Space charge density contours produced by ground corona around a 100-m-tall tower at the moment of the first triggered CG flash 23:47 UT. The contour lines correspond to the total charge density in $\mathrm{nC} \mathrm{m}^{-3}$. 

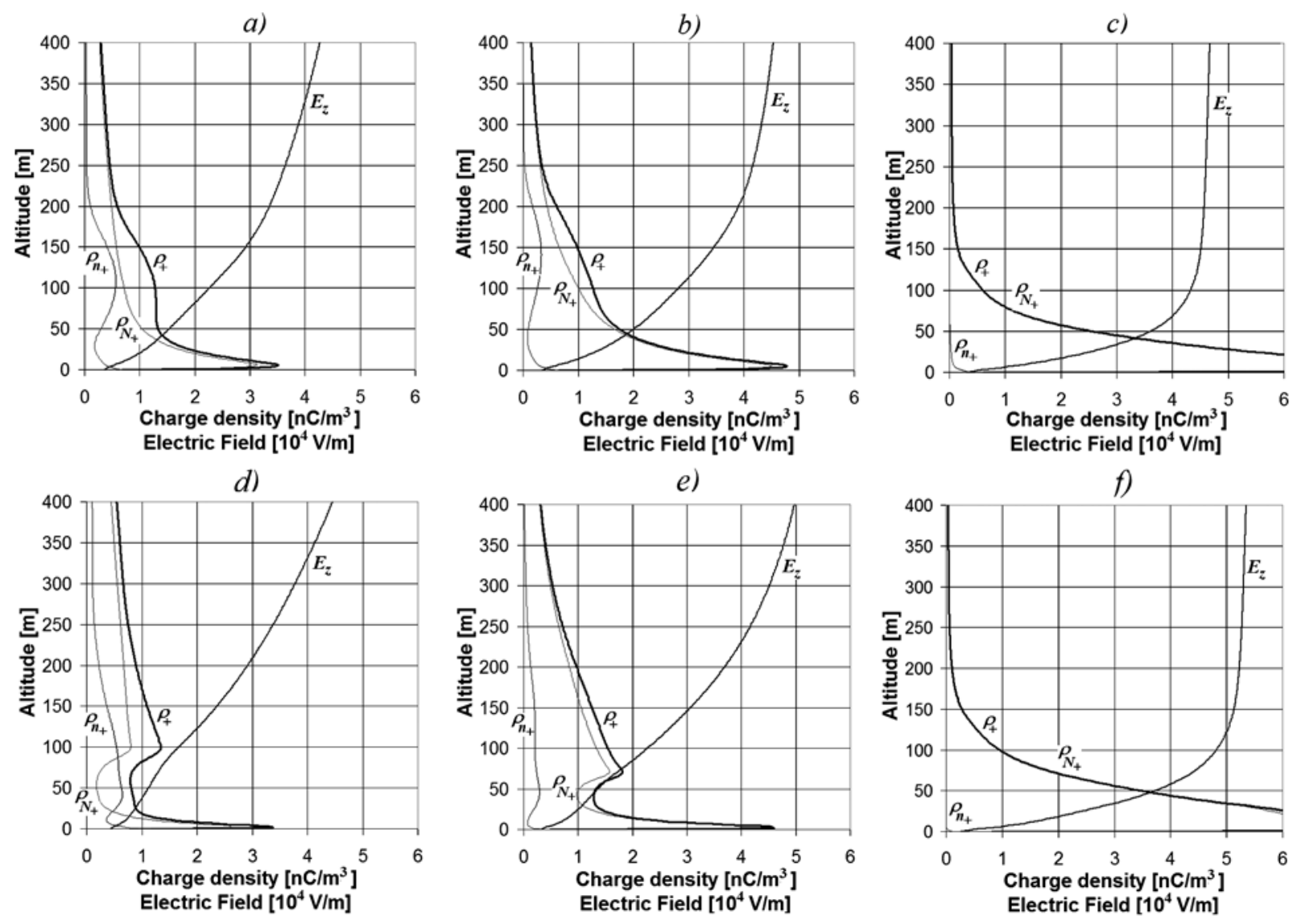

Figure 4. Charge density of positive small ions $\rho_{n+}$, positive large ions $\rho_{N^{+}}$, total charge density $\rho_{+}$, and electric field profiles as a function of altitude for different initial aerosol particle concentrations: (a, d) $5 \times$ $10^{9} \mathrm{~m}^{-3}$; (b, e) $10^{10} \mathrm{~m}^{-3}$, and (c, f) $5 \times 10^{10} \mathrm{~m}^{-3}$ at the moment of the $(\mathrm{a}, \mathrm{b}, \mathrm{c})$ first and $(\mathrm{d}, \mathrm{e}, \mathrm{f})$ second triggered CG flashes. Negative small $\rho_{n-}$ and large $\rho_{N-}$ ion densities are not shown since they are negligible in comparison with the positive ions at the considered instants.

urban area $N_{0}=5 \times 10^{10} \mathrm{~m}^{-3}$. Figure 4 shows the vertical profiles of the space charge density and the electric field at the moment of the first and second triggered CG flashes (23:47, 23:53 UT). Note that when the neutral aerosol particle concentration increases, the attachment of small ions to neutral aerosol particles is enhanced, forming large ions especially close to ground. This result suggests that the small ions cannot drift to high altitudes in sites with high neutral aerosol particle concentrations, creating a thinner and more concentrated space charge layer, in agreement with the predictions of Chauzy and Raizonville [1982] and the measurements of Horii et al. [2006] in the north coast of Japan. This is the case of Figures $4 \mathrm{c}$ and $4 \mathrm{f}$ where the vertical background electric field $E_{z}$ is distorted mostly at low altitudes (lower than about $100 \mathrm{~m}$ ). On the contrary, the small ions drift upward a few hundreds of meters before they get attached into large ions when the neutral aerosol particle concentration is low (Figure 4a). Hence the space charge extends vertically creating a thick layer, distorting also the background field $E_{z}$ at high altitudes as well (several hundreds of meters above ground).

[28] After the first flash, the electric field at ground level changes its polarity (Figure 2) and negative ions are produced by negative corona at the ground surface. Then, the background electric field starts increasing again because of the regeneration of the cloud charge. During this period, the created small negative ions drift upward until they attach to neutral aerosol particles or recombine with large positive ions. Similar to the case of small positive ions, the vertical drift of these small negative ions depends upon the neutral aerosol particle concentration. The thickness of the small negative ion layer decreases as the concentration of neutral aerosol particles increases. Especially for low aerosol particle concentrations (Figure $4 \mathrm{~d}$ and $4 \mathrm{e}$ ), the negative ions drift several tens of meters and recombine with the existing positive large ions, producing a reduction on the total ion density at about $50 \mathrm{~m}$ above ground at the moment of the second flash 23:53 UT. As the thundercloud electric field regenerates, the electric field at ground level becomes positive again after a few minutes. After this, new small positive ions are injected into the layer when the ground electric field reaches the positive corona onset field again, reestablishing the space charge density close to ground to conditions similar to those during the first flash.

[29] It can be observed that the space charge layer significantly distorts the ambient electric field produced by the thundercloud, as it can be seen in Figure 4. The background electric field changes as function of altitude from about the corona onset electric field $E_{0}$ at ground level to the thundercloud electric field at several hundreds of 
a)

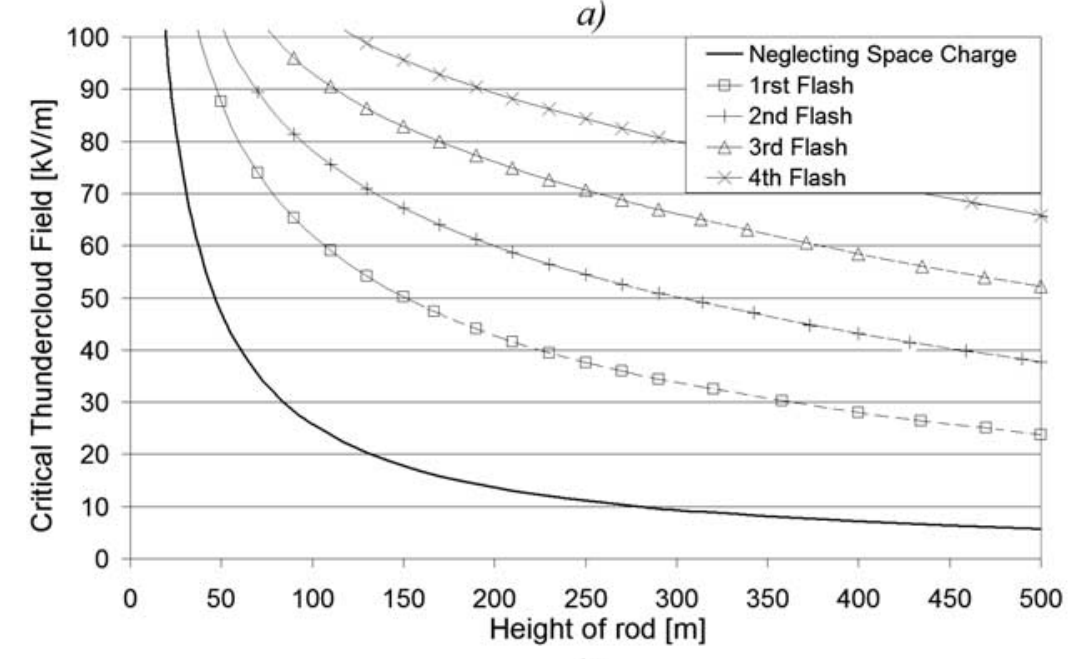

b)

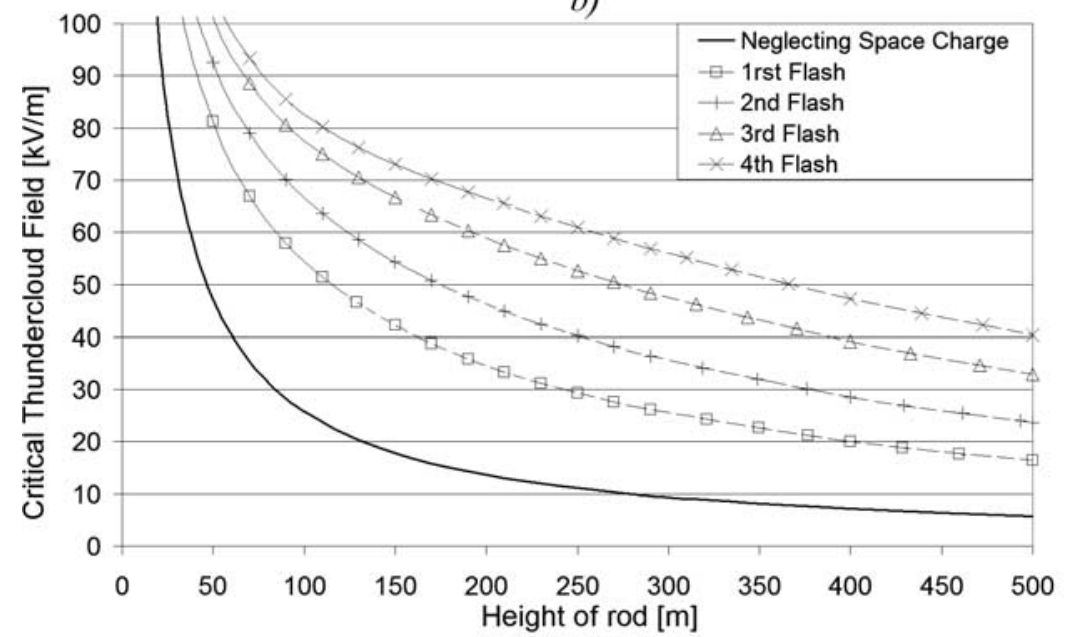

c)

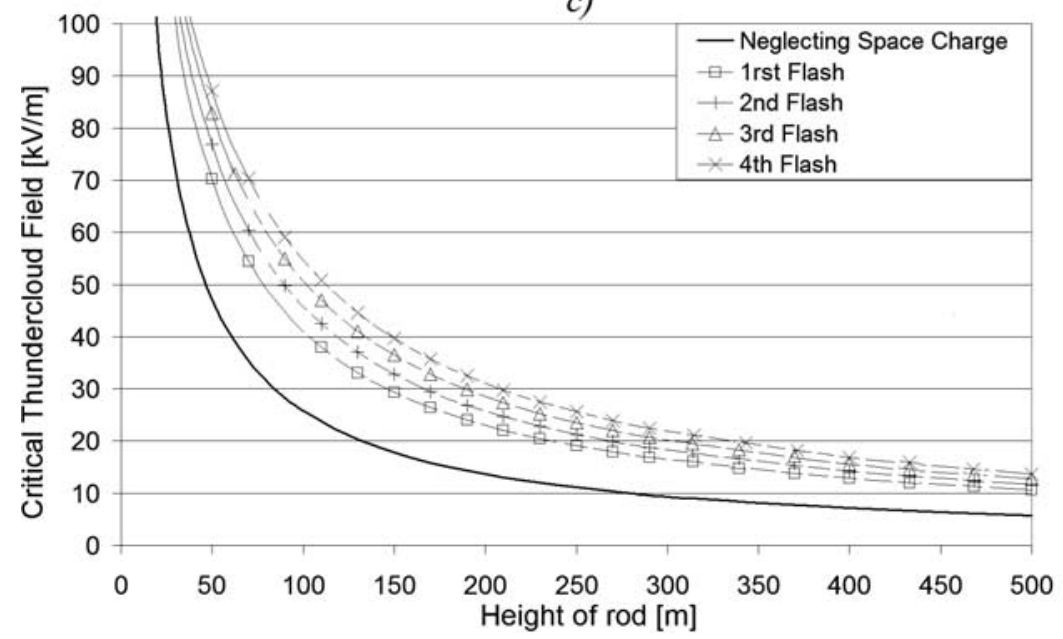

Figure 5. Critical thundercloud electric field $E_{\text {cloud }}^{(\text {crit })}$ required to initiate upward leaders as a function of the tower height, for different initial aerosol particle concentrations: (a) $5 \times 10^{9} \mathrm{~m}^{-3}$, (b) $10^{10} \mathrm{~m}^{-3}$, (c) $5 \times 10^{10} \mathrm{~m}^{-3}$. The critical leader inception electric field computed by neglecting the space charge layer is also shown.

meters above ground [Chauzy and Raizonville, 1982]. Thus, the space charge layer distorts the effective background electric field at which glow corona is produced, and streamers and upward leaders are initiated from the tip of a grounded tower under the influence of the thundercloud electric field. For this reason, the critical thundercloud electric field $E_{\text {cloud }}^{\text {(crit) }}$ required to initiate upward leaders from a tall isolated tower is computed taking into account the obtained space 

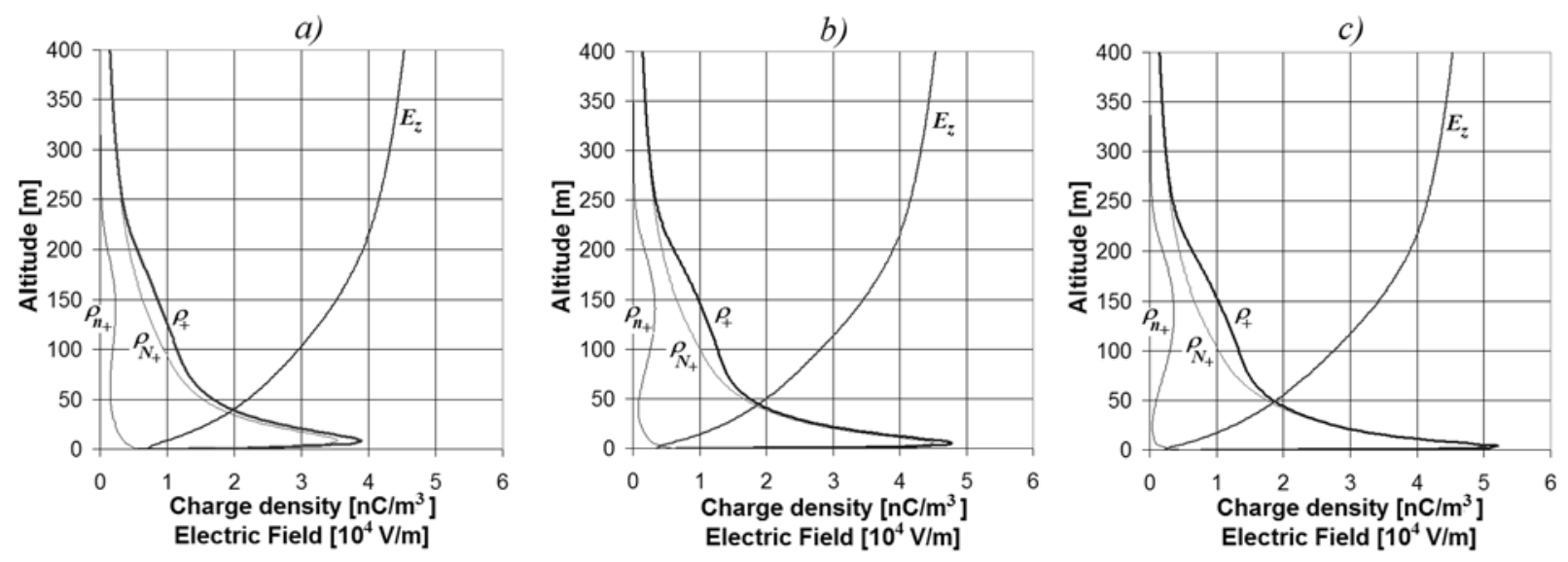

Figure 6. Charge density of positive small ions $\rho_{n^{+}}$, positive large ions $\rho_{N^{+}}$, total charge density $\rho_{+}$, and electric field profiles as a function of altitude for different corona current densities (a) $J_{\mathrm{c}}^{\mathrm{KSC}} / 10$, (b) $J_{\mathrm{c}}^{\mathrm{KSC}}$, and (c) $10 \times J_{\mathrm{c}}^{\mathrm{KSC}}$ at the moment of the first triggered $\mathrm{CG}$ flash. The reference current density $J_{\mathrm{c}}^{\mathrm{KSC}}$ corresponds to the values derived for Florida [Soula, 1994].

charge density profiles. Note that the glow corona is not taken into account in the analysis for sake of simplicity but further details about the production of glow corona under the influence of thunderclouds are given in the work of Aleksandrov et al. [2001, 2005].

[30] Figure 5 shows the critical thundercloud electric field $E_{\text {cloud }}^{\text {(crit) }}$ as a function of the tower height for the considered neutral aerosol particle concentrations. As reference value, the field $E_{\text {cloud }}^{\text {(crit) }}$ is also computed by neglecting the space charge layer. As it can be seen in Figure 5, the critical thundercloud electric field $E_{\text {cloud }}^{\text {(crit) }}$ is significantly influenced by the neutral aerosol particle concentration of the site. For a given $C G$ flash, the critical cloud electric fields $E_{\text {cloud }}^{\text {(crit) }}$ required to initiate upward leaders from a grounded tower of a given height are larger when the neutral aerosol particle concentration of the site is low. For instance, the critical cloud electric field computed for a 200-m-tall tower in a site with neutral aerosol particle concentration of $5 \times 10^{9} \mathrm{~m}^{-3}$ (clean rural zone) is about $43 \mathrm{kV} \mathrm{m}^{-1}$, while for a place with high aerosol particle concentration of $5 \times 10^{10} \mathrm{~m}^{-3}$ (moderately polluted urban area) the critical field is only about $23 \mathrm{kV} \mathrm{m}^{-1}$ at the moment of the first flash. In the same way, the minimum height of a tower from which an upward lightning leader is initiated under the influence of a given thundercloud electric field decreases as the aerosol particle concentration of the site increases. At the moment of the first flash, upward leaders are initiated under the influence of a thundercloud electric field of $50 \mathrm{kV} \mathrm{m}^{-1}$ from towers higher than 150,115 , and $78 \mathrm{~m}$ for sites with neutral aerosol particle concentrations of $5 \times 10^{9} \mathrm{~m}^{-3}, 10^{10} \mathrm{~m}^{-3}$, and $5 \times 10^{10} \mathrm{~m}^{-3}$, respectively. This fact clearly suggests that the initiation of an upward lightning from towers (or other tall grounded structures as transmission lines, wind mills, etc.) does not only depends upon the height of the tower but also upon the thundercloud electric fields and the neutral aerosol particle concentration of the site. Thus, upward leaders can be initiated from a tower of a given height under the influence of lower thundercloud electric fields in polluted sites (with high neutral aerosol particle concentration) than in clean rural zones (with low neutral aerosol particle concentration). This result also applies to coastal areas where aerosol particles over the sea are carried inland by wind, as in the case of the coast of Japan [Sunaga and Shindo, 2005]. Interestingly, field observations in Japan suggest that upward flashes occur even from structures of moderate height (lower than 50-m tall and located on flat terrain) in winter [Miki, 2006]. This result could be partially explained on the basis of the present analysis considering the high thundercloud activity and the high aerosol particle concentration [Sunaga and Shindo, 2005] in the coast of Japan during winter thunderstorms.

[31] Furthermore, note that the thickness of the space charge layer is reduced and the critical thundercloud electric fields approach the values obtained in calculations performed by neglecting the space charge layer (Figure 5) as the aerosol particle concentration increases. This condition corresponds to the most critical case where the electric field shielding produced by the space charge layer is negligible or nonexistent. Therefore it corresponds to the lowest values of the thundercloud electric fields required to initiate upward leaders from grounded objects.

[32] On the other hand, we can observe that the critical thundercloud electric fields $E_{\text {cloud }}^{\text {(crit) }}$ also change in the case of multiple flashes to ground, especially for low neutral aerosol particle concentrations. Because of the reduction on the net space charge density produced by the negative ions injected after each flash (Figure 4), the electric field profile during the subsequent flashes differs from the case of the first flash. Because of the fact that the surface electric field is maintained approximately constant by the corona production and the electric field divergence is reduced by the decrease in the total space charge density, the background electric field $E_{z}$ at low altitudes during the subsequent flashes is reduced in comparison with the conditions at the moment of the first flash. Therefore the critical thundercloud electric fields required to initiate upward leaders during the subsequent flashes are larger than in the first flash especially at low neutral aerosol particle concentrations, as it can be seen in Figure 5.

[33] It is important to point out that the results presented above are based on the assumption that the measured electric field [Soula and Chauzy, 1991] used to drive the space charge calculation is representative of a typical 
a)

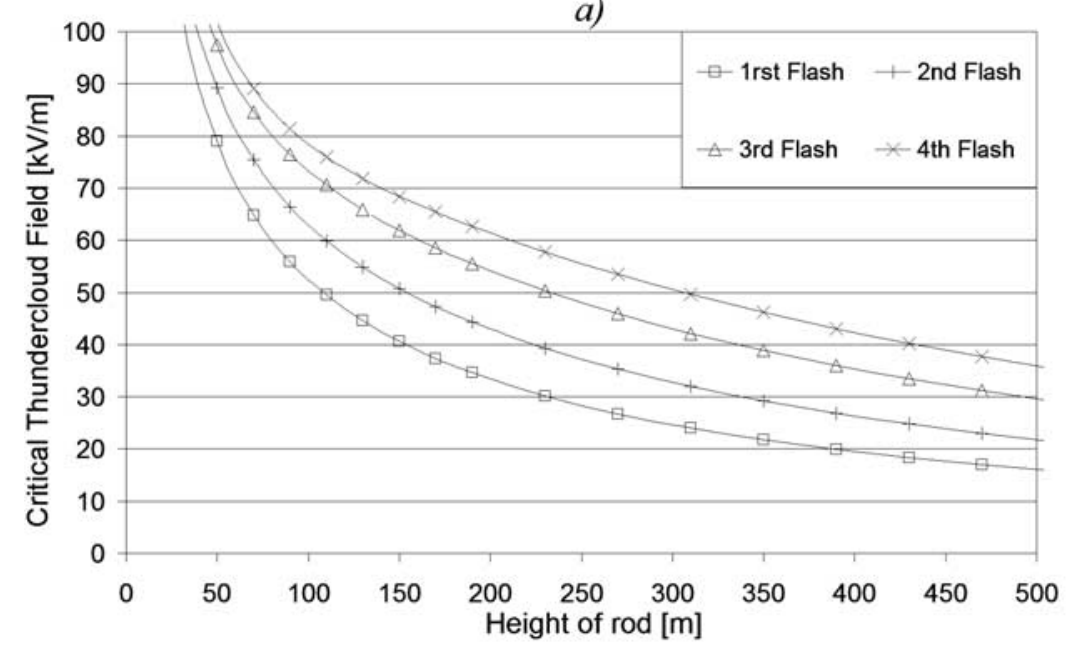

b)

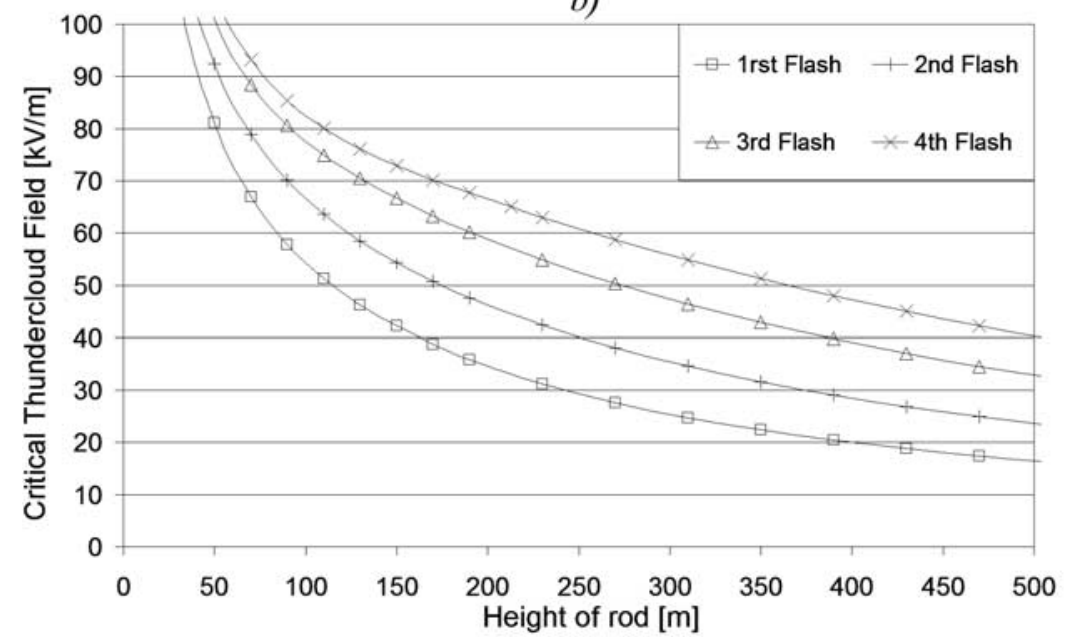

c)

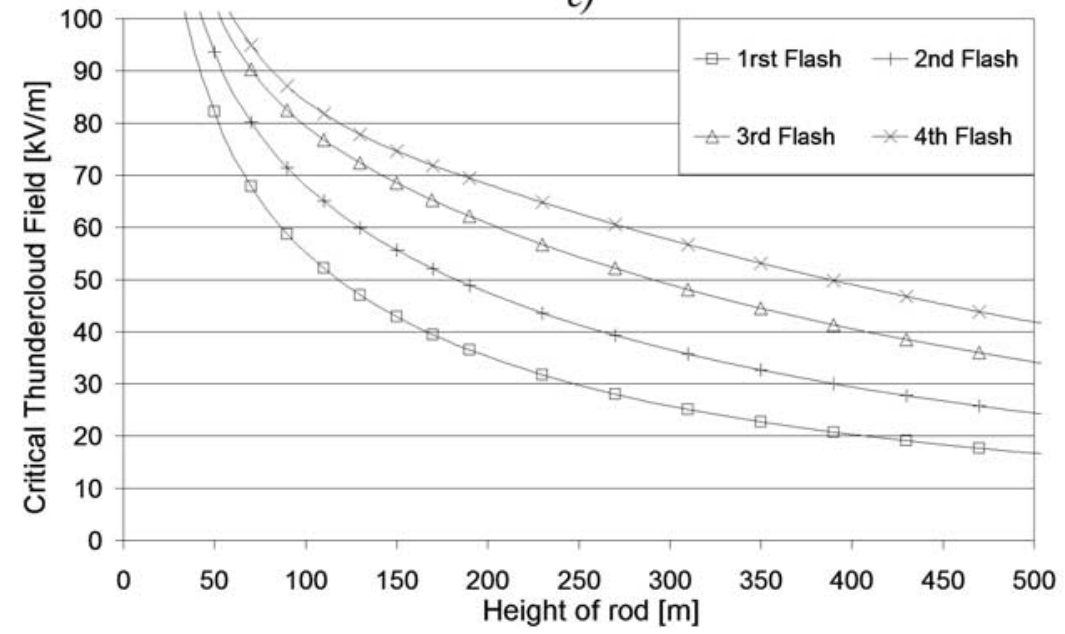

Figure 7. Critical thundercloud electric field $E_{\text {cloud }}^{(\text {crit }}$ required to initiate upward leaders as a function of the tower height, for the corona current densities (a) $J_{\mathrm{c}}^{\mathrm{KSC}} / 10$, (b) $J_{\mathrm{c}}^{\mathrm{KSC}}$, and (c) $10 \times J_{\mathrm{c}}^{\mathrm{KSC}}$ at the moment of the first triggered CG flash.

thundercloud, even for places with high aerosol particle concentration. Since the aerosol particles have an indirect effect on clouds [Penner et al., 2001] they may affect the thundercloud electric field. However, the used electric fields are considered as representative of those produced by a typical thundercloud since similar electric field values have been measured in other experiments [Marshall et al., 1995; Taksuoka et al., 1991]. 


\subsection{Effect of Corona Current Density}

[34] Since the space charge layer is produced by corona discharges from ground irregularities like grass, bushes, and trees, the corona current density is defined by the type of vegetation and surface roughness of the analyzed place [Soula, 1994]. The parameters $a_{1}, a_{2}$, and $a_{3}$ of Equation (8) derived for the corona current density at the Kennedy Space Center in Florida $J_{\mathrm{c}}^{\mathrm{KSC}}[$ Soula, 1994] are used as reference values. Parameters 10 times smaller (applicable to a region with less vegetation and less surface roughness) and 10 times larger (applicable to a region with more vegetation and surface roughness) than the reference values are also considered. These values would result in corona current densities 10 times smaller $J_{\mathrm{c}}^{\mathrm{KSC}} / 10$ and 10 times larger $10 \times J_{\mathrm{c}}^{\mathrm{KSC}}$ for the same ground electric field $E$.

[35] Figure 6 shows the profiles of the space charge density and of the background electric field obtained by changing the corona current density but keeping the neutral aerosol particle concentration constant and equal to $5 \times 10^{9} \mathrm{~m}^{-3}$. As it can be seen, the space charge profile does not vary significantly even though the corona current density is changed by one order of magnitude. In the same way, the background electric field as a function of altitude is not significantly influenced by the change in the corona current density. However, the electric field at the ground changed appreciably, taking values of 7.1, 3.6, and $2.2 \mathrm{kV} \mathrm{m}^{-1}$ for the three current densities considered $J_{\mathrm{c}}^{\mathrm{KSC}} / 10, J_{\mathrm{c}}^{\mathrm{KSC}}$, and $110 \times J_{\mathrm{c}}^{\mathrm{KSC}}$, respectively. This fact shows that a low corona current density is compensated by an increase in the electric field at ground level and vice versa, balancing the production of corona at ground level. Hence the amount of released corona ions (i.e., the space charge density close to ground) in the three cases considered is roughly the same, as it can be seen in Figure 6. Nevertheless, it is noteworthy that the discussion presented above does not apply to the case of smooth water surfaces where no corona effect appears [Chauzy et al., 1989].

[36] We can also note that the critical thundercloud field required to initiate upward leaders is not significantly affected by the considered corona current densities (Figure 7). In the most critical case, that is, when the corona current density is reduced 10 times, only a difference of about $15 \%$ on the critical thundercloud electric field is observed. This result shows that the differences on vegetation (corona current density) have only a minor influence on the effect of the space charge layer on the initiation of upward leaders from tall towers, unlike the suggestion by Golde [1978].

\section{Conclusions}

[37] The critical thundercloud electric field required to initiate self-propagating upward lightning leaders from tall towers is computed by taking into account the space charge layer formed by corona ions produced at ground level but neglecting corona at the tip of the tower. It is found that the space charge layer considerably shields the background electric fields produced by the thundercloud, affecting the conditions required to initiate upward lightning. Furthermore, the influence of different factors such as the electrostatic shielding produced by the tower, the corona current density, and the neutral aerosol particle concentration is evaluated. It is found that the shielding effect of the ground electric field produced by the tower itself inhibits the formation of space charge very close to it, but it does not affect the overall effect of the space charge layer. In a similar way, the corona current density (associated with the irregularities and vegetation of the ground surface of the site) has a slight effect on the conditions required to initiate upward leaders for the cases considered in this study. On the other hand, the space charge layer thickness and the critical thundercloud electric field necessary to initiate upward leaders depend significantly on the neutral aerosol particle concentration of the place where the tower is located. The minimum height of a tower from which upward lightning leaders are initiated when exposed to a given thundercloud electric field decreases as the aerosol particle concentration of the site increases. In other words, upward initiated lightning from tall towers (or other tall grounded structures as transmission lines, wind mills, etc.) requires a lower thundercloud electric field in the case of polluted sites or coastal areas where the neutral aerosol particle concentration is large.

\section{References}

Aleksandrov, N. L., E. M. Bazelyan, R. B. Carpenter, M. M. Drabkin, and Yu. P. Raizer (2001), The effect of coronae on leader inception and development under thunderstorm conditions and in long air gaps, J. Phys. D: Appl. Phys, 34, 3256-3266.

Aleksandrov, N. L., E. M. Bazelyan, M. M. Drabkin, and R. B. Carpenter (2002), Corona discharge at the tip of a tall object in the electric field of a thundercloud, Plasma Phys. Rep., 28, 1032-1045.

Aleksandrov, N. L., Bazelyan, and Y. Raizer (2005), The effect of a corona discharge on a lightning attachment, Plasma Phys. Rep., 31, 75-91.

Becerra, M., and V. Cooray (2006a), A simplified physical model to determine the lightning upward connecting leader inception, IEEE Trans. Power Delivery, 21(2), 897-908.

Becerra, M., and V. Cooray (2006b), Time dependent evaluation of the lightning upward connecting leader inception, J. Phys. D: Appl. Phys, $39,4695-4702$

Berger, K. (1977), The Earth flash, Lightning vol. 1, edited by R. H. Golde, Elsevier, New York.

Chauzy, S., and P. Raizonville (1982), Space charge layers created by coronae at ground level below thunderclouds: Measurements and modeling, J. Geophys. Res., 87(C4), 31431982.

Chauzy, S., and C. Renella (1985), Computed response of the space charge layer created by corona at ground level to external electric field variations beneath a thundercloud, J. Geophys. Res., 90(D4), 6051-6057.

Chauzy, S., and S. Soula (1999), Contribution of the ground corona ions to the convective changing mechanism, Atmos. Res., 51, 279-300.

Chauzy, S., S. Soula, and S. Despiau (1989), Ground corona and lightning, J. Geophys. Res., 94(D11), 13,115-13,119.

Dellera, L., E. Garbagnati, M. Bernardi, A. Bondiou, V. Cooray, I. Gallimberti, A. Pedersen, F. Ruhling (1997), Lightning exposure of structures and interception efficiency of air terminals, CIGRE Report, Task Force 33.01.03.

Golde, R. H. (1978), Lightning and tall structures, Proc. IEEE, 125(4), $347-351$.

Horii, K., K. Nakamura, and S. Ichi Sumi (2006), Review of the experiment of triggered lightning by rocket in Japan, Proceedings 28th International Conference on Lightning Protection ICLP 2006, p. 44-51.

Marshall, T. C., W. Rison, K. Rust, M. Stolzengurg, J. C. Willet, and W. P. Winn (1995), Rocket and balloon observations of electric field in two thunderstorms, J. Geophys. Res., 100(D10), 20,815-20,828.

Miki, M. (2006), Observation of current and leader development characteristics of winter lightning, Proceedings 28th International Conference on Lightning Protection ICLP 2006, p. 14-19.

Penner, J. E., M. Andreae, H. Annegarn, L. Barrie, J. Feichter, D. Hegg, A. Jayaraman, R. Leaitch, D. Murphy, J. Nganga, and G. Pitari (2001), Aerosols, their Direct and Indirect Effects, in Climate Change 2001: The Scientific Basis. Contribution of Working Group I to the Third Assessment Report of the Intergovernmental Panel on Climate Change, edited by J. T. Houghton, Y. Ding, D. J. Griggs, M. Noguer, P. J. van der Linden, X. Dai, K. Maskell, and C. A. Johnson, Cambridge Univ. Press, New York.

Qie, X., S. Soula, and S. Chauzy (1994), Influence of ion attachment on the vertical distribution of the electric field and charge density below a thunderstorm, Ann. Geophys., 12, 1218-1228. 
Rizk, F. (1994), Modeling of lightning incidence to tall structures Part II: Application, IEEE Trans. Power. Delivery, PWRD-9, 172-193.

Roache, P. (1976), Computational Fluid Dynamics, 2nd Ed., Hermosa, Albuquerque, N.M.

Standler, R. B., and W. P. Winn (1991), Effects of coronae on electric fields beneath thunderstorms, Q. J. R. Meteorol. Soc., 105, 2851979.

Soula, S. (1994), Transfer of electrical space charge from corona and thundercloud: Measurements and modeling, J. Geophys. Res., 99(D5), $10759-10765$.

Soula, S., and S. Chauzy (1991), Multilevel measurement of the electric field underneath a thundercloud 1: A new system and the associated data processing, J. Geophys. Res., 96(D12), 22,319-22,326.

Sunaga, Y., and T. Shindo (2005), Influence of space charge on upward leader initiation, IEEJ Trans. PE, 125, 789-796.

Swarztrauber, P., and R. Sweet (1975), Efficient FORTRAN subprograms for the solution of elliptic partial differential equations, NCAR, Tech. Note NCAR-TN/IA-109, p. 135-137.
Taksuoka, K., K. Nakamura, M. Minowa, K. Horii (1991), Electric field measurement by rocket under the thundercloud, Proceedings 7th International Symposium on High Voltage Engineering ISH 1991, paper 81.03. Twomey, S. (1977), Atmospheric Aerosols, Elsevier, New York.

Willet, J. C., D. A. Davis, and P. Laroche (1999), An experimental study of positive leaders initiating rocket-triggered lightning, Atmos. Res., 51, 189-219.

Yializis, A., E. Kuffel, and P. H. Alexander (1978), An optimized charge simulation method for the calculation of high voltage fields, IEEE Trans. Power Appar. Syst., PAS 97(6), 2434-2438.

M. Becerra and V. Cooray, Division of Electricity and Lightning Research, Uppsala University, Uppsala, Sweden. (marley.becerra@angstrom.uu.se)

S. Chauzy and S. Soula, Laboratoire d'Aérologie, Université Paul Sabatier, UMR CNRS 5560, Toulouse, France. 